\title{
Análisis de la gestión del conocimiento en las bibliotecas universitarias de la Comunidad Valenciana: propuesta de un modelo y acciones de mejora
}

\author{
Salvador Sánchez García de las Bayonas \\ Universidad de Alicante (España)
}

\section{Resumen}

Se analiza la situación y la implantación de programas de gestión del conocimiento en las bibliotecas universitarias y académicas, en el marco de la convergencia con el Espacio Europeo de Educación Superior en la Comunidad Valenciana, y se realiza una propuesta de acciones a desarrollar, desde el punto de vista de la gestión organizativa, a través de una encuesta enviada a los directores de los centros implicados, el análisis de la información de sus páginas webs (información institucional, memorias, acceso a recursos, etcétera), así como del uso de sus intranets y de las culturas corporativas con las que trabajan en relación con la importancia dada a compartir el conocimiento entre los colaboradores. La encuesta recoge aspectos como la formación, la gestión de la intranet corporativa, la gestión de las bibliotecas y del personal y el estudio de los recursos informativos. Por otro lado, se examinan los procesos de evaluación llevados a cabo en las bibliotecas universitarias siguiendo el modelo de guía de evaluación establecido en el II Plan de Calidad de las Universidades aprobado por el Real Decreto 408/2001, de 20 de abril del 2001, y cómo han repercutido en la mejora de la gestión del conocimiento. Por último se hace una revisión bibliográfica exhaustiva de la bibliografía profesional disponible y relacionada con el tema en español, catalán e inglés. Toda la información fue presentada en una página web, disponible en el portal de la Fundación Iberoamericana del Conocimiento y actualizada periódicamente con las acciones desarrolladas respecto a la investigación y la información de interés. Los resultados obtenidos son comparados con modelos de referencia presentes en la bibliografía profesional y con el estudio de otros casos prácticos desarrollados tanto en España como en Europa y Estados Unidos, además de mediante el uso de los indicadores, recomendaciones y normas establecidas por organismos oficiales como REBIUN o IFLA, con el fin de establecer un análisis DAFO que determine los puntos fuertes y débiles, así como las amenazas y debilidades. Se observa la existencia de acciones y propuestas específicas relacionadas con la gestión del conocimiento 
con resultados dispares según la biblioteca analizada, pero no existe un modelo común sistemático que haya sido aplicado ni el desarrollo de una cultura de compartir el conocimiento, aunque en todas las encuestas se reconoce su importancia. La falta del desarrollo de los flujos de conocimiento se hace más palpable en el caso del conocimiento tácito, uno de los puntos más débiles en la gestión del conocimiento en las bibliotecas universitarias de la Comunidad Valenciana.

Palabras clave: Bibliotecas universitarias. Gestión del conocimiento. Comunidad Valenciana. Gestión de la información.

\section{Abstract}

This study analyses the current state of university and academic libraries within the framework of the convergence with the European Space of Superior Education in the Region of Valencia along with a proposal of actions to develop, from an organisation management angle and focused on the implementation of knowledge management programmes therein. Research is based on the results of a questionnaire sent out to the heads of the corresponding centres; library websites (institutional information, reports, access to resources, and so on) and the use of their intranets and corporative culture in relation to the importance given to knowledge-sharing between collaborators. The survey gathers aspects like formation, management of the corporative intranet, management of libraries and personnel, and study of the informative resources. Secondly, the evaluation processes carried out in university libraries are examined following the model of guide of evaluation established in the II Plan of Quality of the Universities approved by Real Decree 408/2001, of 20 April 2001, together with their impact on the improvement of knowledge management. Finally, an exhaustive review is carried out on the professional bibliography available on this subject in Spanish, Catalan and English. All of this information is presented on a website that can be accessed from the site of the Fundación Iberoamericana del Conocimiento and is periodically updated with any new activities carried out in this area of research as well as other interesting information on this subject. The results obtained are compared with reference models included in the professional bibliography, the study of other practical cases developed as much in Spain as in Europe and the United States and by using markers, recommendations and norms established by such official bodies as REBIUN (the Spanish University Library Network) or IFLA (the International Federation of Library Associations), with the purpose of establishing an analysis DAFO that determines strong and weak points, as well as threats and weaknesses. It can be seen that specific actions and proposals on knowledge management do exist, although results vary depending on the library. However, it is clear that no common systematic model has been applied nor has a knowledge-sharing culture been devised, despite the fact that all of the 
questionnaires recognise the importance of both. The lack of the development of the knowledge flows becomes more concrete in the case of the tacit knowledge, one of the weak points in the management of the knowledge in the university libraries of the Region of Valencia.

Key words: University libraries. Knowledge management. Region of Valencia. Information management.

\section{Introducción}

\subsection{Espacio Europeo de Educación Superior, universidad española y bibliotecas universitarias}

Desde que en 1998 y 1999 se reunieron en la Sorbona y Bolonia los ministros europeos comunitarios de educación con el fin de establecer un proceso de convergencia entre todas las universidades de la Unión hasta crear un Espacio Europeo de Educación Superior, no más tarde del año 2010, la educación superior está sufriendo las mayores transformaciones vistas desde hace décadas.

Ante este trascendental cambio de las universidades europeas, el mundo bibliotecario universitario español ha tomado cartas en el asunto. Desde hacía tiempo, las bibliotecas universitarias estaban sufriendo una transformación a gran escala motivada por la incorporación de las nuevas tecnologías, por la aparición de nuevos servicios y recursos proporcionados por Internet y por el desarrollo de procesos de digitalización.

Pero ahora es necesario también subir al tren de la convergencia europea. Por esta razón, los máximos representantes de las bibliotecas universitarias de España, en su asamblea general de 2001, celebrada en la Universidad de Alicante, encargaron la elaboración de un plan estratégico para los próximos años que pudiera responder a los nuevos desafíos. El plan fue aprobado en la siguiente asamblea, celebrada en 2002 en Valladolid.

En este plan estratégico se establece una serie de objetivos estratégicos y acciones para desarrollarlos, hoy en día en marcha y con la mayoría de las bibliotecas de las universidades españolas (públicas y privadas) involucradas en mayor o menor medida.

No obstante, sin ningún género de duda, lo más importante de este plan es el desarrollo de una nueva concepción de biblioteca universitaria transformada en lo que se denomina Centro de Recursos para el Aprendizaje y la Investigación (CRAI), teniendo la referencia del modelo anglosajón. Dentro del paradigma de aprendizaje por enseñanza, la biblioteca universitaria pasa de ser un servicio de apoyo a la docencia y a la investigación a constituir un centro de recursos disponibles para el desarrollo del aprendizaje del propio usuario, ya sea para el estudio o para la 
investigación. Los entornos digitales y virtuales están contribuyendo, en gran medida, a esta concepción.

\subsection{La gestión del conocimiento en las bibliotecas universitarias}

Los objetivos de la gestión del conocimiento en las bibliotecas universitarias deben ir íntimamente ligados a los objetivos estratégicos de la propia universidad. Desvincularlos supone un preludio del fracaso. Uno de los aspectos evaluados en todos los procesos de análisis de la calidad en las bibliotecas universitarias es la integración en el marco institucional, en el contexto del plan estratégico, la relación con la docencia y la investigación acometida en la universidad (Guía, s. f.).

Por otro lado, toda programación de objetivos en bibliotecas universitarias debe enmarcarse dentro del plan estratégico de REBIUN 2003-2006 (1), si quiere participar en el concierto de todas las bibliotecas universitarias a nivel del Estado español.

La gestión del conocimiento eficiente en las bibliotecas universitarias desde el punto de vista de la gestión corporativa debe aprovechar todos los conocimientos tácitos y explícitos, los individuales y corporativos, los internos y externos a las bibliotecas a través de las siguientes líneas estratégicas: $a$ ) establecer una cultura corporativa de "compartición" del conocimiento (o lo que en el mundo anglosajón se conoce como knowledge sharing); b) mejora del control de la producción científica de la universidad, tanto de las tesis doctorales como de los archivos abiertos (OAI), etcétera; $c$ ) creación de repositorios de conocimiento (o bases de datos de conocimiento) para la gestión eficaz; d) explicitación del máximo conocimiento tácito posible en cuanto a gestión por medio de intranets corporativas, manuales de procedimiento, herramientas para compartir conocimiento entre los colaboradores de la biblioteca, etcétera; $e$ ) mayor proyección de la imagen corporativa, del trabajo en colaboración a nivel institucional de la universidad, a nivel local, nacional e internacional; $f$ ) desarrollo de las competencias de los colaboradores de la biblioteca universitaria a través, fundamentalmente, de procesos selectivos eficientes y de acciones formativas acordes con las competencias necesarias para el desempeño de las funciones.

Si la materia prima de la universidad es el conocimiento, la biblioteca universitaria es el lugar en donde se recopila, organiza, procesa, mantiene y difunde el conocimiento. Por lo tanto, el valor estratégico es evidente. De la gestión más o menos eficaz de este servicio derivará el mayor o menor aprovechamiento de su materia prima (el conocimiento), y, por lo tanto, de su productividad y rentabilidad académica, docente e investigadora.

Por otro lado, si la gestión del conocimiento tiene como pilares fundamentales la gestión de la información y la gestión de los recursos humanos, en las bibliotecas universitarias están instalados esos pilares. Si de algo saben los biblio- 
tecarios es precisamente de gestionar la información. De hecho, es tanta la experiencia en la gestión de este recurso que la propia REBIUN pretende, en su línea estratégica número 1, convertir la biblioteca universitaria en un sistema de recursos para la planificación de un sistema de información único para toda la universidad. Tanto a nivel de desarrollo de los procesos como de atención a los usuarios, la biblioteca universitaria es el principal foco de la gestión de la información. Desde una interpretación propia de la teoría de la comunicación, podemos entenderla como la recopilación, la organización, el mantenimiento y la difusión, dentro de un contexto, de los datos representados en documentos, con el fin de transmitir significados que los usuarios transformarán en conocimiento. Davenport y Prusak (1998) hablan de acceso, organización, fijación y transferencia del conocimiento.

Pero, a pesar de la gran experiencia, surgen retos importantes analizados muy pobremente: si esto es lo que se entiende por gestión de la información, solo podrán contribuir las bibliotecas universitarias a la gestión de la "información explícita”. Deberían, sin embargo, buscarse fórmulas para gestionar la "información tácita", es decir, la información más estratégica, para explicitarla. Es un auténtico reto: en un futuro, las bibliotecas universitarias deberán gestionar foros de discusión, encuentros entre estudiantes y profesores fuera de las aulas, encuentros entre estudiantes, personal docente e investigador y profesionales de la información, crear bases de datos de apuntes, desarrollar servicios de referencia digitales más allá del mero correo electrónico (chat, videoconferencias, etcétera).

\subsection{Situación de las bibliotecas universitarias de la Comunidad Valenciana}

El sistema universitario Valenciano depende de la Conselleria de Cultura, Educación y Deportes a través de la Dirección General de Universidades e Investigación. Cuenta con cinco universidades públicas (2) y una privada (3).

Además, la Comunidad Valenciana cuenta con la presencia de sedes de otras universidades ajenas al sistema universitario valenciano, como son la sede en Valencia de la Universitat Oberta de Catalunya, una sede de la Universidad Internacional Menéndez Pelayo y un centro asociado a la UNED, entre otras.

Siempre ha existido una gran tradición universitaria en la Comunidad Valenciana, siendo la Universitat de València - Estudi General el más antiguo de los centros (fundado en 1499) y el más numeroso de toda España en cuanto a usuarios potenciales de las bibliotecas, después de la UNED y seguida de cerca por la Universidad Complutense de Madrid (4).

Cada una de las universidades valencianas cuenta con su propio servicio de bibliotecas perteneciente a la Red de Bibliotecas Universitarias (REBIUN), dependientes, en su mayor parte, de los vicerrectorados de Investigación.

Scire. $13: 2$ (jul.-dic. 2007) 17-40. ISSN 1135-3716. 
Las fichas técnicas resumidas de cada servicio de bibliotecas son las siguientes (5):

\begin{tabular}{|c|c|c|c|}
\hline Centro & Estructura organizativa & Extensión & Recursos \\
\hline $\begin{array}{l}\text { Universidad } \\
\text { de Alicante }\end{array}$ & $\begin{array}{l}\text { Una dirección académica } \\
\text { y otra técnica; dividida } \\
\text { en unidades centrales y } \\
\text { bibliotecas de centro; } \\
1 \text { campus con dos salas } \\
\text { de lectura en sedes } \\
\text { por la provincia }\end{array}$ & $\begin{array}{l}19579 \mathrm{~m}^{2} \text { y } 427468 \\
\text { volúmenes para } \\
34061 \text { usuarios } \\
\text { potenciales (6) }\end{array}$ & $\begin{array}{l}\text { Unas } 169 \text { b. d. } \\
\text { disponibles; unas } 4018 \\
\text { revistas electrónicas } \\
\text { accesibles desde } 18 \\
\text { portales contratados }\end{array}$ \\
\hline $\begin{array}{l}\text { Universidad } \\
\text { Cardenal Herrera }\end{array}$ & $\begin{array}{l}\text { Una biblioteca central } \\
\text { estructurada por } \\
\text { unidades y servicios }\end{array}$ & $\begin{array}{l}5200 \mathrm{~m}^{2} \text { y } 75521 \\
\text { volúmenes para unos } \\
7234 \text { usuarios }\end{array}$ & $\begin{array}{l}\text { Unas } 29 \text { b. d. } \\
\text { disponibles; acceso } \\
\text { a unas } 36 \text { revistas } \\
\text { electrónicas }\end{array}$ \\
\hline $\begin{array}{l}\text { Universidad } \\
\text { Miguel Hernández }\end{array}$ & $\begin{array}{l}5 \text { sedes en } 4 \text { campus } \\
\text { de distintas localidades } \\
\text { de la provincia } \\
\text { de Alicante }\end{array}$ & - & $\begin{array}{l}\text { Algo más de } 20 \text { b. d. } \\
\text { disponibles; } \\
\text { unas } 2000 \text { revistas } \\
\text { electrónicas } \\
\text { accesibles desde } 18 \\
\text { portales contratados }\end{array}$ \\
\hline $\begin{array}{l}\text { Universitat de València } \\
\text { - Estudi General }\end{array}$ & $\begin{array}{l}\text { Una dirección académica } \\
\text { y otra técnica; dividida } \\
\text { en servicios centrales } \\
\text { y bibliotecas de centro } \\
\text { distribuidas en } 3 \text { campus }\end{array}$ & $\begin{array}{l}34688 \mathrm{~m}^{2} \text { y } 1128945 \\
\text { volúmenes para unos } \\
108678 \text { usuarios }\end{array}$ & $\begin{array}{l}\text { Unas } 86 \text { b. d. } \\
\text { disponibles; unas } 3125 \\
\text { revistas electrónicas } \\
\text { accesibles desde } 14 \\
\text { portales contratados }\end{array}$ \\
\hline $\begin{array}{l}\text { Universitat Politècnica } \\
\text { de Valéncia }\end{array}$ & $\begin{array}{l}\text { Estructura basada } \\
\text { en las bibliotecas } \\
\text { de centro distribuidas } \\
\text { por seis localidades de la } \\
\text { Comunidad Valenciana }\end{array}$ & $\begin{array}{l}11896 \mathrm{~m}^{2} \text { y } 366269 \\
\text { volúmenes para } \\
\text { unos } 44116 \text { usuarios }\end{array}$ & $\begin{array}{l}\text { Unas } 68 \text { b. d. } \\
\text { disponibles. Acceso } \\
\text { a unas } 2947 \text { revistas } \\
\text { electrónicas }\end{array}$ \\
\hline $\begin{array}{l}\text { Universitat Jaume I } \\
\text { de Castellón }\end{array}$ & $\begin{array}{l}\text { Estructura basada } \\
\text { en los procesos técnicos; } \\
\text { no existen bibliotecas } \\
\text { de centro; } 1 \text { campus } \\
\text { (Riu Sec) }\end{array}$ & $\begin{array}{l}8000 \mathrm{~m}^{2} \text { y } 251960 \\
\text { volúmenes para unos } \\
17132 \text { usuarios }\end{array}$ & $\begin{array}{l}\text { Unas } 53 \text { b. d. } \\
\text { disponibles; } \\
\text { unas } 3427 \text { revistas } \\
\text { electrónicas } \\
\text { accesibles desde } 12 \\
\text { portales contratados }\end{array}$ \\
\hline
\end{tabular}

El signo - indica que no se han encontrado datos en los anuarios REBIUN. 


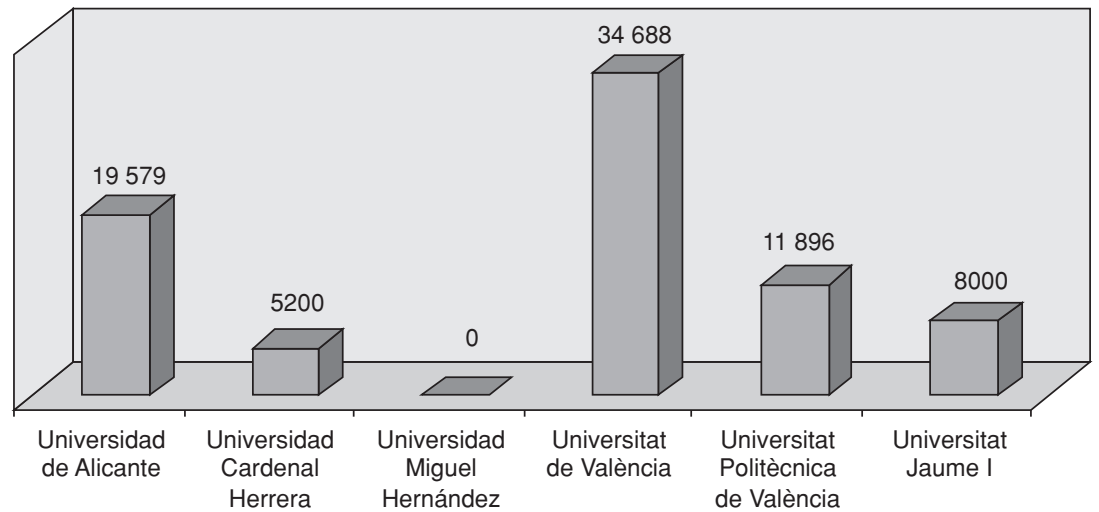

Figura 1. Comparación de las instalaciones de los servicios de biblioteca en $\mathrm{m}^{2}$ de superficie.

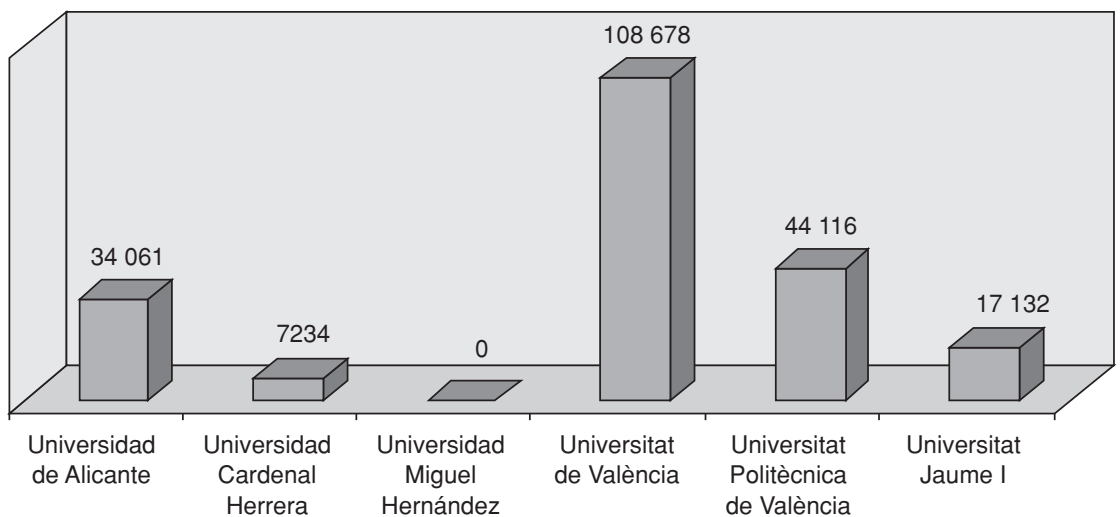

Figura 2. Comparación de los usuarios potenciales de los servicios de biblioteca.

\begin{tabular}{|l|c|}
\hline \multicolumn{2}{|l|}{$\begin{array}{c}\text { Las “Normas y directrices para bibliotecas universitarias y científicas" recomiendan } \mathbf{1} \mathbf{~ m}^{2} \text { / usuario } \\
\text { con carácter general. Los datos de esta tabla son los reflejados en el Anuario REBIUN 2003 }\end{array}$} \\
\hline Universidad de Alicante & $0,57 \mathrm{~m}^{2} /$ usuario \\
\hline Universidad Cardenal Herrera & $0,72 \mathrm{~m}^{2} /$ usuario \\
\hline Universidad Miguel Hernández & No hay datos en el Anuario REBIUN 2003 \\
\hline Universitat de València & $12,7 \mathrm{~m}^{2} /$ usuario \\
\hline Universitat Politècnica de València & $15,2 \mathrm{~m}^{2} /$ usuario \\
\hline Universitat Jaume I de Castellón & $13,6 \mathrm{~m}^{2} /$ usuario \\
\hline
\end{tabular}

Tabla I. Indicadores de REBIUN: $\mathrm{m}^{2}$ / usuario potencial.

Scire. 13 : 2 (jul.-dic. 2007) 17-40. ISSN 1135-3716. 
Las "Normas y directrices para bibliotecas universitarias y científicas" recomiendan una colección mínima de $\mathbf{5 0} 000$ volúmenes y el incremento de 1 volumen por estudiante y 10 por PDI al año. Los datos de esta tabla son los reflejados en el Anuario REBIUN 2003

\begin{tabular}{|l|c|}
\hline Universidad de Alicante & 0,8 vols. / usuario \\
\hline Universidad Cardenal Herrera & 1,3 vols. / usuario \\
\hline Universidad Miguel Hernández & No hay datos en el Anuario REBIUN 2003 \\
\hline Universitat de València & 0,3 vols. / usuario \\
\hline Universitat Politècnica de València & 0,7 vols. / usuario \\
\hline Universitat Jaume I de Castellón & 0,9 vols. / usuario \\
\hline
\end{tabular}

Tabla II. Indicadores de REBIUN: incremento de monografías / usuarios potenciales.

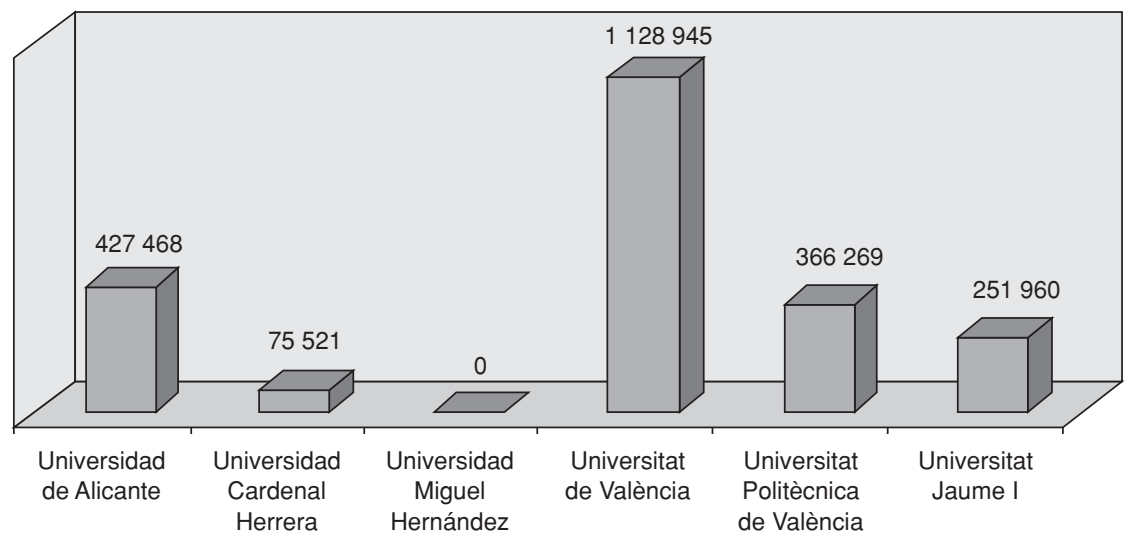

Figura 3. Comparación de los fondos de los servicios de biblioteca.

Del análisis podemos extraer varias conclusiones:

1. Las estructuras de los servicios son muy dispares, respondiendo cada una de ellas a las características propias de la universidad (varios campus, tamaño, presupuesto, etcétera). Así, encontramos servicios de bibliotecas estructurados exclusivamente por bibliotecas de centro - como la Universitat Politècnica de València—, por unidades y servicios sin bibliotecas de centro — la Universidad Cardenal Herrera - o por procesos — la Universitat Jaume I de Castellón—. Las realidades son totalmente distintas en cada servicio de bibliotecas.

2. La disponibilidad de recursos electrónicos, como indicador tecnológico del servicio de bibliotecas, es muy dispar entre las universidades. Mientras que en unas solo existen una treintena de bases de datos contratadas disponibles, en otras se su-

Scire. 13 : 2 (jul.-dic. 2007) 17-40. ISSN 1135-3716. 
peran las 160. El servicio recibido no es similar entre los usuarios de los distintos servicios. Una de las razones esgrimidas para esta disparidad es la inexistencia de un consorcio entre las bibliotecas para la adquisición cooperativa de los recursos. La reducción del coste o la maximización del disponible sería mayor al aumentar la capacidad de negociar con los distribuidores, entre otros factores. Ha habido experiencias aisladas en compras cooperativas de recursos, pero no existe una formalización de este proceso. Desde la Comunidad Valenciana se observan con interés consorcios como el CBUA (Consorcio de Bibliotecas Universitarias de Andalucía) y el MADROÑO (Consorcio de las Universidades de la Comunidad de Madrid y de la UNED para la Cooperación Bibliotecaria), pero, sobre todo, el ejemplo del CBUC (Consorci de Bibliotèques Universitaries de Catalunya) (Anglada, 2004). Algunas bibliotecas universitarias se han planteado unirse a él; otras, crear el propio consorcio para las bibliotecas de Valencia, y otras, esperar al consorcio que surja del plan estratégico de REBIUN.

3. Las universidades de mayor tamaño cumplen con las recomendaciones de REBIUN, debido al gran número de usuarios potenciales. Es el caso de la Universitat de València, donde solo hay 0,31 metros cuadrados por usuario potencial. La más cercana a la recomendación es la Universidad Cardenal Herrera $\left(0,74 \mathrm{~m}^{2} /\right.$ usuario), y la más alejada, la Universitat Politècnica de Valencia $\left(0,10 \mathrm{~m}^{2} /\right.$ usuario). Este contraste lo marca, sin duda, la diferencia en el número de usuarios.

La conclusión extraída es la evidente falta de inversiones en infraestructuras y edificios destinados a bibliotecas.

En relación con los fondos, todas las universidades de la Comunidad Valenciana cumplen con creces con los mínimos establecidos. Sin embargo, viendo las cifras resultantes, deducimos aspectos muy interesantes: las universidades de mayor tamaño (Universitat de València y Universitat Politècnica de València) presentan las mayores deficiencias en cuanto a fondos. La distribución entre los usuarios no es adecuada. El más de un millón de fondos de la Universitat de Valencia se distribuye a 9,09 volúmenes por usuario potencial, por ejemplo.

\section{Metodología}

\subsection{Cuestionario: descripción y motivación}

Durante los meses de abril y mayo se remitió a los directores o responsables de los servicios de biblioteca de las universidades de la Comunidad Valenciana un cuestionario para analizar distintos aspectos relacionados con la gestión del conocimiento corporativo. En total se enviaron seis formularios (uno por cada servicio de biblioteca) por correo electrónico para contestar a preguntas agrupadas en otros seis puntos focales:

Scire. 13 : 2 (jul.-dic. 2007) 17-40. ISSN 1135-3716. 
Descripción y características del servicio de bibliotecas

— Tipología de los colaboradores: personal fijo, contratado, etcétera

- Análisis cuantitativo de los colaboradores implicados y de los usuarios atendidos por el servicio de bibliotecas

- Tamaño del servicio de bibliotecas

Aspectos relacionados con la formación de los colaboradores

— Existencia de planes de formación específicos y generales

- Número de acciones formativas

- Existencia de planes de inducción y orientación

Desarrollo de la gestión de la intranet corporativa

- Uso de la intranet

- Procedimiento para la introducción y modificación de contenidos

Aspectos relacionados con la gestión bibliotecaria y de personal dentro

del servicio de bibliotecas

- Existencia de planes de gestión del conocimiento y acciones relacionadas

- Expectativas sobre la gestión del conocimiento

- Existencia de planificación estratégica

- Análisis de la cultura corporativa del servicio de bibliotecas

- Existencia de planes de comunicación

- Estabilidad y rotación de los colaboradores

- Participación en grupos de trabajo multidisciplinares

Aprovechamiento de los recursos y servicios

- Maximización del acceso a los recursos y servicios

Por último, un capítulo de sugerencias y comentarios

Las preguntas fueron seleccionadas según otros estudios precedentes sobre la gestión del conocimiento en unidades similares, como el realizado por el equipo de Anna Valls, de la Universitat Politècnica de Catalunya (Valls y Hernández, 2003), y de indicadores establecidos por REBIUN en sus estadísticas anuales, relacionadas directamente con la gestión del conocimiento.

\subsection{Análisis de las páginas webs de las bibliotecas universitarias y de sus intranets}

En el estudio de las páginas webs oficiales de los servicios de biblioteca de las distintas universidades se analizaron los siguientes puntos: $a$ ) accesibilidad desde la página principal de la universidad: cuántos clics son necesarios para acceder, en qué nivel aparecen $\left(1 .^{\circ}, 2 .^{\circ}\right.$, etcétera); visibilidad del contenido desde cualquier navegador (se usaron Internet Explorer 6.0, Netscape 4.5 y Mozilla Firefox); $b$ ) cantidad y calidad del contenido: desarrollo de la información institucional (directorios, memorias, estadísticas, documentos estratégicos, etcétera), acceso a múltiples recursos, desarrollo de servicios y recursos on-line, etcétera; actualidad de la información: cada cuánto tiempo se actualiza por el webmaster, correcto funcionamiento de los enlaces, etcétera.

Scire. $13: 2$ (jul.-dic. 2007) 17-40. ISSN 1135-3716. 
El estudio de las intranets corporativas fue menos exhaustivo debido a la imposibilidad material de tener acceso a todas las disponibles. Para su análisis se tuvo en cuenta, casi en su totalidad, la información proporcionada por los directores o responsables de los distintos servicios de biblioteca en el cuestionario remitido.

\subsection{Procesos de evaluación}

En la actualidad, la mayoría de los servicios de biblioteca del Estado español se encuentran dentro de procesos de evaluación, en el marco del II Plan de Evaluación de la Calidad de las Universidades (Guía, s. f.). La documentación al respecto es numerosa, y una buena fuente para consultar los distintos procesos de evaluación puede ser la página de formación de REBIUN.

Distintos servicios de biblioteca de universidades de la Comunidad Valenciana se encuentran en este trance, tan importante como paso previo para la realización de planificaciones estratégicas y, más globalmente, para adentrarse en el marco del Espacio Europeo de Educación Superior.

Analizamos en este estudio algunos de estos procesos, unos ya concluidos y otros en desarrollo, desde el enfoque de la gestión del conocimiento. Pretendíamos averiguar cómo la gestión de la calidad puede ayudar a mejorar la gestión del conocimiento en los servicios de bibliotecas y, por otro lado, cómo la gestión del conocimiento puede mejorar la calidad de los servicios y recursos de estas mismas instituciones.

La conclusión fue que es la mejor gestión del conocimiento la que incrementa la calidad de los servicios y recursos de las bibliotecas universitarias de la Comunidad Valenciana. Allí donde se aprovecha el conocimiento corporativo, añadiendo valor a la gestión bibliotecaria, la calidad es mayor, y así lo reflejan los informes finales de evaluación.

Los procedimientos normalizados y documentados (explicitados), la menor rotación de personal (menor coste en formación y mayor aprovechamiento del conocimiento individual o tácito), los análisis de la satisfacción y de las necesidades de los usuarios, etcétera, repercuten en un mejor servicio de bibliotecas.

\subsection{Revisión bibliográfica}

La documentación recopilada ha sido sobre todo en inglés, debido a la escasa producción de información en español o de traducciones al castellano de autores no españoles (Nonaka y Takeuchi, por ejemplo).

Por otro lado, las fuentes de información bibliográfica y documental consultadas han sido las más representativas del mundo de las bibliotecas universitarias, como REBIUN (Plan Estratégico 2003-2006; "Normas y directrices para bibliotecas universitarias y científicas", etcétera), OCLC o páginas oficiales de la Unión Europea donde se tratara, en particular, la integración en el marco del Espacio Europeo de Educación Superior.

Scire. 13 : 2 (jul.-dic. 2007) 17-40. ISSN 1135-3716. 
Dentro de esta revisión bibliográfica se prestó atención tanto a los aspectos teóricos sobre la gestión del conocimiento como a las experiencias de casos prácticos relacionados con las bibliotecas universitarias o científicas y, por último, a la descripción y análisis de la situación de las bibliotecas universitarias y científicas, y en concreto de la gestión del conocimiento en las mismas.

\subsection{Página web de la investigación}

Para el desarrollo y difusión de la investigación se elaboró una página web con toda la información relacionada: objetivos, universidades y centros objetos del estudio, modelo de cuestionario utilizado, bibliografía relacionada, enlace a la página oficial de IBERSID 2004, resultados y enlaces de interés. También se daban a conocer las últimas noticias surgidas durante el desarrollo del estudio.

\section{Resultados}

\subsection{Descripción objetiva de los resultados}

\subsubsection{Descripción y características de la universidad}

En este primer capítulo de la encuesta se pretendían obtener fundamentalmente dos informaciones: por un lado, cuál era el tamaño tanto del servicio de biblioteca analizado como de la universidad, en general, donde se enmarcaba, y, por otro lado, el grado de rotación no funcional de los colaboradores tanto del servicio de biblioteca como de toda la universidad.

Se parte de la premisa de que, a mayor eventualidad de los colaboradores por rotaciones no funcionales, mayor es también el nivel de fuga de conocimiento, sobre todo tácito, dentro de la organización, y viceversa.

Los servicios bibliotecarios de las universidades de la Comunidad Valenciana podrían clasificarse como de tamaño medio-pequeño, según los indicadores de personal destinado y número de bibliotecas o unidades de servicio. Las cifras oscilan entre el único punto bibliotecario (biblioteca o unidad) de la Universidad Cardenal Herrera y los más de 10 de la Universitat de València.

Los resultados indican, por otro lado, que el nivel de estabilidad de los colaboradores, en general, es alto. Por ejemplo, en la Universitat de València, la más numerosa en cuanto a personal (210 colaboradores destinados al Servei de Biblioteques), el 71,4\% son funcionarios de carrera. En el lado opuesto se encuentra la Universidad de Alicante, que no llega al 50\% de personal fijo.

\subsubsection{Formación}

En el capítulo de formación, pretendíamos averiguar la importancia dada a este punto por los servicios de biblioteca, si el desarrollo del mismo era el adecuado y si la incorporación de nuevos colaboradores era tenida en cuenta.

Scire. 13 : 2 (jul.-dic. 2007) 17-40. ISSN 1135-3716. 
El resultado obtenido indica una clara apuesta por la formación en los servicios de biblioteca. En la mayoría de ellos existe un plan de formación, más o menos desarrollado, enmarcado dentro de los planes de formación generales de toda la universidad, como principal garantía de su éxito.

Sin embargo, no hay planes de inducción, orientación o tutorización en la mayoría de las ocasiones. Se ha detectado la entrega de manuales básicos direccionales, algunas visitas guiadas y pocas acciones concretas más, que, sin duda, no constituyen una garantía suficiente para la adecuada incorporación de nuevos colaboradores.

\subsubsection{Gestión de la intranet corporativa}

El objetivo era averiguar el grado de implantación de la intranet como herramienta tecnológica para la gestión del conocimiento, su desarrollo en cuanto al mantenimiento, su uso y el contenido incorporado.

En la mayoría de los casos existe una intranet corporativa implantada.

El número de personas encargadas de su mantenimiento y actualización (webmasters) es reducido, siendo un caso atípico el de la Universitat de València, donde 8 personas tienen licencia para modificar el contenido de la intranet.

No es habitual la figura de un moderador o animador, con el consiguiente poco aprovechamiento del recurso.

Sin embargo, parece claro, para la mayoría de los servicios de biblioteca, que las intranets corporativas no son solo un almacén de documentación. También se usan para cuestiones de comunicación, punto de encuentro e informativo.

\subsubsection{Gestión bibliotecaria y de personal}

Pretendíamos determinar la existencia de planes de gestión del conocimiento, averiguar la concienciación de su necesidad para la mejora de los servicios, recursos y procedimientos, saber cuáles eran las expectativas sobre la gestión del conocimiento y, por último, valorar la cultura corporativa sobre "compartir conocimiento" (sharing knowledge).

No son habituales los planes de gestión del conocimiento específicos en los servicios de biblioteca de las universidades. En algunos casos, se limitan tan solo al desarrollo de las actividades formativas (como es el caso de la Universitat de València).

A pesar de esta deficiencia, todos los servicios de biblioteca reconocen la necesidad de su existencia y de los beneficios que pueden aportar tanto para los procesos como para los servicios.

Más claros parecen los cauces para la comunicación interna de los colaboradores, predominando las reuniones (para la comunicación ascendente y horizontal) y comunicados (para la comunicación descendente).

Scire. $13: 2$ (jul.-dic. 2007) 17-40. ISSN 1135-3716. 
Resulta evidente la falta de un conocimiento exacto de cómo aplicar un programa de gestión del conocimiento por parte de los colaboradores de los servicios de biblioteca. Aunque nadie niega sus positivas repercusiones, no parece estar claro cuál es su alcance y trascendencia. Se evidencia la falta de una cultura para compartir el conocimiento, la mayor de las limitaciones.

\subsubsection{Aprovechamiento de los recursos}

Este punto se centraba en el desarrollo tecnológico para el avance de la gestión del conocimiento y su grado de implantación.

En general, el desarrollo de la infraestructura informática es excelente. En la mayoría de los casos existe un campus virtual preparado para acceder a los recursos y servicios de las bibliotecas. La mayoría de las funciones relacionadas con una buena gestión del conocimiento, como la comunicación corporativa, la búsqueda de información, el mantenimiento de bases de datos y el trabajo en equipo cuentan con la infraestructura necesaria.

Sin embargo, la falta de una cultura de "compartición" del conocimiento en la mayoría de los servicios da como resultado una infrautilización de los recursos tecnológicos disponibles.

\subsection{Comparación con indicadores REBIUN}

En este aspecto encontramos dificultades significativas para poder analizar el grado de implantación de la gestión del conocimiento en los servicios de biblioteca. Ninguno de los indicadores establecidos por REBIUN responde con concreción a los necesarios para el análisis de esta circunstancia. Los aportados solo ayudan a comparar el desarrollo general de todos los servicios bibliotecarios de las universidades españolas.

Hacemos una exposición solo para comprobar y corroborar datos ya ofrecidos anteriormente, comparando los servicios bibliotecarios de las universidades de la Comunidad Valenciana con la media general de los participantes en REBIUN.

Los indicadores y los datos han sido extraídos del anuario publicado por REBIUN en 2003, siendo seleccionados aquellos más relacionados con la materia de estudio o con los representativos de la situación de cada servicio de bibliotecas.

Scire. 13 : 2 (jul.-dic. 2007) 17-40. ISSN 1135-3716. 


\begin{tabular}{|l|c|c|c|c|c|c|c|}
\hline & REBIUN & UA & UCH & UMH & UVEG & UPV & UJI \\
\hline$m^{2}$ / usuario & 0,58 & 0,57 & $\mathbf{0 , 7 2}$ & - & 0,32 & 0,27 & 0,47 \\
\hline Monografías / usuario & 1,0 & 0,8 & $\mathbf{1 , 3}$ & - & 0,3 & 0,7 & 0,9 \\
\hline Revistas / investigador & 1,5 & 1,2 & 1,3 & - & 1,3 & 0,2 & 1,4 \\
\hline Informatización / total fondos & $85 \%$ & $\mathbf{1 0 0} \%$ & - & - & $71 \%$ & $53 \%$ & $\mathbf{8 5} \%$ \\
\hline Préstamos / usuarios & 7,6 & 6,6 & 5,4 & - & 3,1 & $\mathbf{1 0 , 2}$ & 7,1 \\
\hline Consultas b. d. / investigador & 155 & $\mathbf{2 0 5}$ & - & - & $\mathbf{2 9 9}$ & 24 & - \\
\hline Artículos electrónicos / investigador & 46 & 20 & - & - & 19 & 27 & - \\
\hline Visitas páginas web / usuario & 86 & 12 & - & - & 22 & $\mathbf{1 4 8}$ & 85 \\
\hline Consultas catálogo / usuario & 47 & 45 & - & - & - & 41 & - \\
\hline Usuarios / personal & 333 & 270 & $\mathbf{3 4 4}$ & - & $\mathbf{6 1 4}$ & $\mathbf{4 6 4}$ & $\mathbf{5 0 4}$ \\
\hline Personal técnico / total colaboradores & $38 \%$ & $33 \%$ & $19 \%$ & - & $36 \%$ & $31 \%$ & $26 \%$ \\
\hline Becarios / total colaboradores & $17 \%$ & $6 \%$ & $14 \%$ & - & $0 \%$ & $6 \%$ & $0 \%$ \\
\hline $\begin{array}{l}\text { Gasto recursos electrónicos / } \\
\text { total gastos }\end{array}$ & $22 \%$ & $21 \%$ & $\mathbf{2 5 \%}$ & - & $16 \%$ & $\mathbf{3 2 \%}$ & $14 \%$ \\
\hline
\end{tabular}

El signo - indica que no se han encontrado datos.

Ponemos las cifras en negrita cuando el servicio bibliotecario supera o iguala la media de REBIUN y en cursiva cuando está por debajo.

\subsection{Análisis DAFO}

\begin{tabular}{|c|c|c|c|}
\hline Puntos fuertes & Puntos débiles & Oportunidades & Amenazas \\
\hline $\begin{array}{l}\text { Es generalizada la } \\
\text { importancia dada a la } \\
\text { formación continua de } \\
\text { los colaboradores. } \\
\text { Existe una rotación no } \\
\text { funcional moderada- } \\
\text { baja de los } \\
\text { colaboradores, siendo } \\
\text { la tendencia a la baja. } \\
\text { Poca dispersión de los } \\
\text { puntos de servicio } \\
\text { bibliotecario. Están } \\
\text { cercanos a los } \\
\text { usuarios. } \\
\text { La mayoría de los } \\
\text { servicios de biblioteca } \\
\text { se encuentran en } \\
\text { procesos de } \\
\text { evaluación. }\end{array}$ & $\begin{array}{l}\text { Falta generalizada de } \\
\text { planes de inducción, } \\
\text { orientación o } \\
\text { tutorización. } \\
\text { A pesar de la poca } \\
\text { dispersión, sí que } \\
\text { faltan puntos de } \\
\text { servicios bibliotecarios. } \\
\text { Sería necesario } \\
\text { disponer de más } \\
\text { bibliotecas de centro o } \\
\text { puntos de lectura. } \\
\text { Inexistencia de planes } \\
\text { estratégicos de los } \\
\text { servicios bibliotecarios, } \\
\text { quizá por la falta de } \\
\text { planes estratégicos } \\
\text { generales de las } \\
\text { universidades o por no } \\
\text { haber concluido los } \\
\text { procesos de } \\
\text { evaluación. }\end{array}$ & $\begin{array}{l}\text { Integración de las } \\
\text { universidades } \\
\text { españolas y, en } \\
\text { concreto, de las de la } \\
\text { Comunidad } \\
\text { Valenciana, en el } \\
\text { Espacio Europeo de } \\
\text { Educación Superior. } \\
\text { Procesos de } \\
\text { evaluación con } \\
\text { introducción de la } \\
\text { filosofía de calidad } \\
\text { total de los servicios y } \\
\text { recursos y las } \\
\text { oportunidades de } \\
\text { mejora posteriores. } \\
\text { Los servicios } \\
\text { bibliotecarios como } \\
\text { centros neurálgicos de } \\
\text { gestión del } \\
\text { conocimiento en las } \\
\text { universidades. }\end{array}$ & $\begin{array}{l}\text { Graves problemas de } \\
\text { financiación. } \\
\text { Poco desarrollo de la } \\
\text { cultura de fomento y } \\
\text { "compartición" del } \\
\text { conocimiento. }\end{array}$ \\
\hline
\end{tabular}




\begin{tabular}{|l|l|l|l|}
\hline Puntos fuertes & Puntos débiles & Oportunidades & Amenazas \\
\hline La mayoría participa & & Cultura y tradición de & \\
en redes cooperativas & & trabajo cooperativo a & \\
como REBIUN, Xarxa & & todos los niveles. & \\
Joan Lluís Vives, & & Nuevo desarrollo & \\
etcétera & normativo universitario & \\
Existe la infraestructura & & generalizado y su & \\
tecnológica necesaria & & implicación en los & \\
para el desarrollo de la & & servicios bibliotecarios. & \\
Gestión del & & \\
Conocimiento. & & & \\
\hline
\end{tabular}

\section{Recomendaciones para la gestión del conocimiento en las bibliotecas universitarias: objetivos estratégicos y acciones}

Dentro de la línea estratégica básica de este estudio, y con el fin de crear un entorno favorable a la gestión del conocimiento y su "compartición", las bibliotecas universitarias pueden plantearse los siguientes objetivos y llevar a cabo las acciones para su consecución de esta forma:

Objetivo 1: cambiar la cultura organizativa para fomentar el intercambio de conocimiento

\section{Acciones}

1. Crear canales informales basados en la confianza y la cooperación.

2. Establecer grupos de investigación y trabajo multidisciplinares, del tipo cluster sectorial, para el desarrollo de servicios, procesos y recursos.

3. Crear foros de discusión en lugar de organizar las reuniones formales, que necesitan tiempo y recursos para su organización y de las que solo se obtiene un "acta" final, pero no "capturas" de conocimiento (Cornella, 2003, p. 9) como condensación de lo aprendido, del nuevo conocimiento modificador de una actitud en la biblioteca universitaria.

4. Adoptar técnicas basadas en $a$ ) la gestión por competencias, $b$ ) la dirección por objetivos, $c$ ) evaluar el desempeño, $d$ ) la motivación por la naturaleza del trabajo, fomentando los reconocimientos más que las recompensas, y $e$ ) establecer planes de carrera.

5. Implicación de todo el personal en los procesos de cambio. La gestión del conocimiento en instituciones como las bibliotecas universitarias suele tropezar con problemas como culturas muy burocratizadas, resistentes al cambio, estrictas en su normativa y procedimientos, etcétera. Realizar cambios en estas condiciones pasa por involucrar al máximo número de colaboradores. 
Objetivo 2: gestionar la motivación, la inspiración y la casualidad

Acciones

1. Creación de espacios de encuentro formativo entre profesores y alumnos donde los recursos informativos y los servicios bibliotecarios tengan una presencia relevante. Tal como recomienda la OCLC en su informe (7), mediante el uso y las posibilidades que ofrecen los course management systems o aplicaciones de software que gestionen los elementos electrónicos de los cursos, workshops, etcétera, donde "la información y el conocimiento puedan ser creados" (8). En este sentido, lo habitual es que la experiencia en KM de los bibliotecarios no haya sido aprovechada.

2. El nuevo modelo de biblioteca defendido por REBIUN como Centro de Recursos para el Aprendizaje y la Investigación (CRAI) ayudará a crear espacios de encuentro y a fomentar la implicación de todos los colectivos de las universidades en la propia gestión del servicio de bibliotecas.

Objetivo 3: mejor control de la producción científica de la propia universidad, para hacerla más accesible a los usuarios de las bibliotecas

Acciones

1. Creación de archivos abiertos en las universidades productoras (OAI). En la actualidad, el sometimiento del mundo científico y académico a los intereses comerciales de las empresas editoriales es exagerado. Muchos investigadores "pagan" por el "privilegio" de publicar. Los archivos abiertos supondrían un control académico y legal fuera de las imposiciones comerciales. Se facilitaría el acceso gratuito a otros investigadores. Sin embargo, habrá que superar problemas técnicos bibliotecarios, suscitar la confianza de los investigadores (que sus publicaciones cuenten para su currículo), adoptar softwares, etcétera.

2. Elaboración, comercialización y explotación de más bases de datos de las tesis doctorales en las universidades. Un ejemplo muy relevante es el TDX de las universidades catalanas. En la actualidad, las tesis doctorales, como productos principales de la creación del conocimiento en la universidad, están infravaloradas e infrautilizadas. Desde REBIUN parece haberse tomado en serio esta situación y ya forma parte de su plan estratégico crear un portal de acceso a los textos completos de las tesis doctorales publicadas en universidades españolas en modo OAI, aprovechando las experiencias precisamente de TDX y la Universidad Complutense de Madrid. El Ministerio de Educación y Ciencia tendrá mucho que decir a este respecto, sobre todo en lo referente a la base de datos TESEO.

Scire. $13: 2$ (jul.-dic. 2007) 17-40. ISSN 1135-3716. 
3. Sistemas de control y gestión del fenómeno denominado serendipidad, mediante la gestión del aprendizaje ensayo-error, por ejemplo.

4. Creación de spin-offs: viveros de empresas o de iniciativas empresariales o comerciales. Las bibliotecas universitarias cuentan con una base inestimable y un puesto de partida traducido a ventaja competitiva para el desarrollo de productos y servicios que pueden ser comercializados en el mundo académico. Algunas iniciativas de la Universitat Oberta de Catalunya y otras universidades, tanto españolas como europeas y americanas, ya han demostrado su viabilidad y rentabilidad. Las bibliotecas universitarias también pueden emprender acciones en busca de financiación: vender el software para servicios de noticias, bases de datos de sumarios electrónicos, competir con los distribuidores, cobrar por el mantenimiento de sus servicios. La autofinanciación es una respuesta clara al problema presupuestario.

Objetivo 4: creación de repositorios de conocimiento para la gestión eficaz

\section{Acciones}

1. La creación de un directorio de expertos es muy útil para poder solucionar problemas de la gestión del día a día, así como de las acciones de planificación estratégica y operativa. Debe haber dos tipos de directorio: uno interno, de expertos de la biblioteca universitaria, al estilo de las páginas amarillas elaboradas en la Universitat Politècnica de Catalunya por el equipo en el que participa Anna Valls (2003), y otro de expertos externos a la biblioteca, que recoja a los más destacados en cada temática bibliotecaria o documental, así como las empresas del sector, recursos informativos de interés, best practices, etcétera. La plataforma ideal para esta herramienta es, sin duda, la intranet corporativa.

2. Elaboración de bases de datos que registren los e-mails, formularios webs utilizados, reclamaciones y sugerencias, etcétera, recogidos en cualquiera de los servicios, secciones, unidades, etcétera, de la biblioteca universitaria. Un ejemplo de buena práctica es el que se desarrolla en estos momentos en la Universitat Oberta de Catalunya con este tipo de material. Allí se trabaja con los e-mails cruzados entre estudiantes y profesores, entre los miembros de los foros, etcétera, desde una perspectiva sobre todo docente. El modelo a implantar debería adaptarse a las necesidades y actividades de gestión y de servicios propias de la biblioteca universitaria.

3. Creación de repositorios de objetos de aprendizaje. Asimismo, se debe proporcionar los medios, el entorno y la formación a los docentes para que elaboren sus propios productos o herramientas docentes. Un ejemplo muy claro es el caso de La Factoría, en la Universitat Politècnica de Catalunya.

Scire. 13 : 2 (jul.-dic. 2007) 17-40. ISSN 1135-3716. 
Objetivo 5: proyección de la imagen corporativa de la biblioteca universitaria, así como del trabajo cooperativo y de colaboración con el entorno bibliotecario y universitario a nivel local, autonómico, estatal e internacional

Acciones

1. Participación en congresos, jornadas, workshops, exposiciones, etcétera, no solo como asistentes, sino también como ponentes. Se difundirían los conocimientos creados en el entorno de la biblioteca universitaria y se participaría en un entorno de "compartición" e intercambio de conocimientos creados en otras instituciones de similares características.

2. Implicación en las actividades cooperativas organizadas por asociaciones u organismos como REBIUN, asociaciones profesionales, etcétera.

Objetivo 6: desarrollo de las capacidades, habilidades, aptitudes y actitudes de los colaboradores de las bibliotecas universitarias en todas las vertientes necesarias mediante el desarrollo de un plan de formación institucional Acciones

1. Elaboración de un catálogo de competencias necesarias para el desarrollo de las funciones en los distintos puestos de la biblioteca universitaria. Para ello es conveniente guiarse por las recomendaciones de organismos como la ALA (2003), REBIUN (9), la Unión Europea (10) o por el catálogo desarrollado por el sistema universitario de Catalunya (2003). Una medida necesaria es representar estas competencias y las acciones de formación necesarias para desarrollarlas en las relaciones de puestos de trabajo de las universidades.

2. Asistencia a acciones formativas externas a la biblioteca universitaria (cursos, seminarios, jornadas, congresos, encuentros, conferencias, etcétera). Una vez realizada la acción formativa, los conocimientos deben ser transferidos al resto de colaboradores mediante "capturas" de conocimiento, elaboración de informes, memorias, etcétera.

3. Desarrollo de la formación on-line. La oferta de cursos o acciones formativas a través de internet es muy variada y de calidad excelente en muchos casos. Motivar al personal a participar en ellas, así como facilitar el desarrollo de la acción, ahorra a la biblioteca universitaria desplazamientos, dietas, viajes, etcétera.

4. Elaboración de materiales multimedia formativos, tanto para el personal como para todos los miembros de la comunidad universitaria. Ejemplos interesantes son el "Tutorial de habilidades informacionales" elaborado por las bibliotecas de la Universidad de Texas y traducido al español por el Servei de Biblioteques i Documentació de la Universitat Politècnica de Catalunya (11).

Scire. 13 : 2 (jul.-dic. 2007) 17-40. ISSN 1135-3716. 
5. Acciones formativas internas con los propios colaboradores de la biblioteca universitaria, dentro del marco formativo general de la Universidad. La formación interna en las organizaciones tiene una ventaja: los asistentes la aceptan con facilidad y no la ven como una imposición.

6. Desarrollo de un plan de inducción de nuevos colaboradores. La incorporación de nuevos profesionales necesita un período de adaptación, de toma de contacto con la realidad con la que se enfrenta. Es necesario reducir el tiempo para esta adaptación y aprovechar eficazmente el que sea necesario usar. Un ejemplo desarrollado de gran interés es el plan de inducción desarrollado en la biblioteca universitaria de la Universitat Politècnica de Catalunya (Valls y Hernández, 2003).

7. Desarrollo de un plan de retención del conocimiento de los colaboradores que abandonan la biblioteca universitaria. Las prejubilaciones y jubilaciones, las rotaciones de personal no funcionales, los ajustes de plantilla y las ofertas laborales de mayor interés para los colaboradores deben estar controlados. Perder un colaborador sin retener su conocimiento es perder capital intelectual de la organización.

\section{Conclusiones}

1. Se ha detectado una auténtica falta de cultura organizativa favorable a compartir el conocimiento, a pesar de la consideración de su importancia por parte de todos los servicios de biblioteca. Esto se deduce de los datos recogidos del cuestionario y, más concretamente, de una pregunta formulada de forma directa en la que se confesaba esta deficiencia. Por lo tanto, se trata del principal punto de trabajo para cualquier gestor del conocimiento. Sin esta transformación, las demás acciones carecerán de sentido y se verán abocadas al fracaso.

2. Es patente el desconocimiento de las técnicas propias de gestión del conocimiento por parte de los gestores de los servicios de biblioteca. No se sabe exactamente en qué consisten ni cómo aplicarlas. En algunos casos se confunden con el desarrollo de meras acciones formativas.

3. Existe la infraestructura tecnológica necesaria para el desarrollo de las acciones de gestión del conocimiento pero está infrautilizada, como ocurre en el caso de las intranets con la falta de moderadores, por ejemplo.

4. No hay desarrollados modelos de gestión del conocimiento en servicios bibliotecarios universitarios claros, con su eficacia y eficiencia demostrada, excepto experiencias elogiables como en el caso de la Universitat Politècnica de Catalunya. Es necesario acudir a modelos de gestión del co- 
nocimiento aplicados a instituciones y organismos académicos más globales o que desarrollen servicios educativos de distinta índole.

5. A pesar de las dificultades, los servicios de biblioteca de las universidades son viveros muy oportunos y adecuados para el desarrollo de la gestión del conocimiento corporativo. Pocas instituciones recogen tantos recursos y entornos propicios para ello. Trabajar con el conocimiento como materia prima fundamental, en un entorno universitario favorable a la creación del mismo, con profesionales entrenados y acostumbrados a actuar en entornos intensivos en información y en constante cambio, son propiedades básicas no apreciables en otras instituciones. Solo es necesario un cambio cultural profundo.

6. En el caso concreto de los servicios bibliotecarios de la Comunidad Valenciana, sería conveniente concienciar a directores y gestores universitarios de toda índole, y a quienes estén relacionados con ellos, de la necesidad de fomentar la iniciativa con respecto al resto del Estado español. El empuje comprometido de REBIUN sería un revulsivo importante, pero no es la justificación para esperar a que otros sean los primeros en hacerlo. De hecho, en los entornos bibliotecarios de las universidades catalanas ya se está trabajando desde hace tiempo.

7. Otro de los recursos positivos para la gestión del conocimiento es la tradición de trabajo cooperativo propio de los servicios bibliotecarios en las universidades españolas, y en concreto en las de la Comunidad Valenciana. Este es un punto fuerte. Sin embargo, es necesario desarrollar más los aspectos cooperativos como las compras consorciadas, por ejemplo. La gestión del conocimiento corporativa y cooperativa conlleva una mayor eficiencia de los recursos.

8. El desarrollo de una cultura de "compartición" del conocimiento pasa, inevitablemente, por la adopción de las técnicas de gestión cooperativa más adecuadas y reseñadas en el punto 4 del objetivo 1. Es importante un cambio en la gestión. El éxito de todo programa de gestión del conocimiento, en cualquier institución, siempre pasa por dos aspectos claves: el apoyo decidido de la dirección (tanto la del servicio de bibliotecas como la de la universidad en general) y la implicación del mayor número de colaboradores de la organización.

\section{Notas}

(1) REBIUN (Red de Bibliotecas Universitarias) es el máximo órgano representativo de las bibliotecas universitarias españolas, y sus actividades, desde el año 1988, han sido trascendentales para su desarrollo. Se trata del organismo referente más destacado de todo el Estado español y servicios tan importantes como el préstamo interbibliotecario,

Scire. $13: 2$ (jul.-dic. 2007) 17-40. ISSN 1135-3716. 
el desarrollo de las colecciones, el acceso a la información electrónica, etcétera, se han afianzado gracias a las pautas, recomendaciones y normas reguladoras de REBIUN y del espíritu cooperativo y de trabajo en grupo que han inculcado en el mundo bibliotecario universitario.

(2) Universidad de Alicante, Universidad Miguel Hernández, Universitat Jaume I de Castellón, Universitat Politècnica de València y Universitat de València - Estudi General.

(3) Universidad Cardenal Herrera.

(4) Dato extraído del Anuario REBIUN 2003.

(5) Datos actualizados el 24/05/2004 y extraídos de las memorias de las bibliotecas universitarias, de los datos de sus páginas webs oficiales y del Anuario REBIUN del año 2003.

(6) Incluye todos los colectivos miembros de la comunidad universitaria: estudiantes, PDI y PAS.

(7) Puede verse el informe de la OCLC (Libraries and the enhancement of e-learning) en la URL http://www5.oclc.org/downloads/community/elearning.pdf (consultado el 12/01/2004).

(8) Traducción al castellano de una frase del mismo informe.

(9) Informe de 2002 del equipo de trabajo de REBIUN "Recursos Humanos" (http://www.crue.org/web-rebiun/GtRecHumInf2001.PDF) y "Competencias del Bibliotecario Digital”, según la línea estratégica 2 para 2003 del plan estratégico.

(10) Relación de eurocompetencias en información y documentación, dentro del proyecto DECIDoc del programa Leonardo da Vinci.

(11) http://bibliotecnica.upc.es/tilt/intro/splash.htm

\section{Referencias}

Agència per a la Qualitat del Sistema Universitari de Catalunya (2003). Habilitat i competències. // Marc general per a la integració europea. Barcelona: Agència de Qualitat Universitària, 2003. 47.

ALA (1991). Guidelines for Behavioral Performance of Reference and Information Services Professionals. // Reference \& User Services Quarterly. 36 (1996) 200-203. http://www.ala.org/rusa (2004-05-19).

Anglada i de Ferrer, Lluís M. a (2004). Cooperación entre bibliotecas universitarias españolas y la experiencia del CBUC. http://internet.cervantes.es/internetcentros/biblioteca/ Coobiblioestambul/ponencia_esp_llan.htm (2004-05-30).

Anuario de las bibliotecas universitarias y científicas españolas 2003 (REBIUN). Madrid: Servicio de Biblioteca de la Universidad Autónoma de Madrid, d. 1. 2000. http://bibliotecnica.upc.es/Rebiun/nova/publicaciones/informeEstadistico03.pdf (200405-30).

Bogliolo, Domenico (2000). Knowledge management in Italian university libraries. Roma: The American University of Rome (Distinguished Lecture Series), 2000. http://www.uniroma1.it/Documentation/GeorgeATesoro.html (2004-05-30).

Scire. 13 : 2 (jul.-dic. 2007) 17-40. ISSN 1135-3716. 
Canals Parera, Alfons; Pérez Gutiérrez, Mario (2001). Hacia la gestión del conocimiento. // La Vanguardia, 27 de abril de 2001. http://www.uoc.edu/web/esp/art/uoc/canals/ canals_imp.html (2004-01-20).

Carta Magna de las Universidades Europeas. http://www.crue.org/cmue.htm.

Cornella, Alfons (2003). Ley del alquimista. // Papeles de Infonomia. 17 (2003) 9.

Davenport, Thomas H.; Prusak, Lawrence (1998). Working knowledge: how organizations manage what they know. Cambridge: Harvard Business School Press, 1998.

Fowler, Rena K. (1998). The university library as learning organization for innovation: an exploratory study. // College and Research Libraries. 59:3 (1998) 220-231.

Guía de evaluación de bibliotecas (s. f.). II Plan de la Calidad de las Universidades, s. f.

La integración del sistema universitario español en el Espacio Europeo de Enseñanza Superior: documento marco (2003). Madrid: Ministerio de Educación, Cultura y Deporte, 2003. http://www.eees.ua.es/conferencias/Documento-Marco_10_Febrero.doc.

Ley Orgánica 6/2001, de 21 de diciembre, de Universidades. http://www.crue.org/pdf/ LOU/LOU.pdf.

Libraries and the enhancement of e-learning (2003). Ohio: OCLC, 2003. http://www5.oclc.org/ downloads/community/elearning.pdf (2004-01-12).

Middleton, Michael (1999). De la gestión de la información a la gestión del conocimiento: perspectivas sobre el desarrollo. // El Profesional de la Información. 8:5 (1999) 10-17.

Moscoso, Purificación (2003). La nueva misión de las bibliotecas universitarias ante el Espacio Europeo de Enseñanza Superior. // Jornadas REBIUN 2003: Los centros para recursos del aprendizaje y la investigación en los procesos de innovación docente. Madrid, 2003. http://biblioteca.uam.es/documentos/Jornadas_REBIUN/4\%20-\%20nueva_ mision_bibliotecas.pdf (2004-05-19).

Nonaka, Ikujiro; Takeuchi, Hirotaka (1995). The Knowledge-Creating Company: how Japanese companies create the dynamics of innovation. Oxford [etc.]: Oxford UP, 1995.

Obeso, Carlos (2000). Información no estructurada. // Infonomia.com, mensaje n. ${ }^{\circ} 2$ (enviado el 20 de julio de 2000).

El papel de las universidades en la Europa del conocimiento - Comisión de las Comunidades Europeas (2003). Bruselas: Comisión de las Comunidades Europeas, 2003. http://www.uah.es/universidad/espacio_europeo/documentos/papel_las\%20_univ_ europa_conocimiento\%20febrero_2003.pdf (2004-01-20).

REBIUN. Red de Bibliotecas Universitarias Españolas (1997). Normas y directrices para bibliotecas universitarias y científicas. Documento de trabajo - Conferencia de Rectores de las Universidades Españolas (CRUE), mayo 1997.

REBIUN. Plan Estratégico de Rebiun 2003-2006. http://www.crue.org/rebiun-plan 2003-2006.htm.

REBIUN. http://aps.unirioja.es/biblio/recursos? $\operatorname{cod} 2=6 \& \operatorname{cod} 1=A \& \operatorname{dir}=$ tipo $\&$ sub $=2$ (2004-07-07).

Shanhong, Tang (2000). Gestión del conocimiento en las bibliotecas del siglo XXI. // IFLA Council and General Conference. 66 (2000). http://www.ifla.org/IV/ifla66/papers/ 057-110s.htm (2004-07-11).

Scire. $13: 2$ (jul.-dic. 2007) 17-40. ISSN 1135-3716. 
Tardón, Eugenio (1999). Bibliotecas universitarias y gestión del conocimiento. // El Profesional de la Información. 8:4 (1999) 22-28.

Tissen, René; Andriessen, Daniel; Lekanne Deprez, Frank (2000). El valor del conocimiento para aumentar el rendimiento en las empresas. Madrid [etc.]: Prentice, 2000.

Valls Pasola, Anna; Hernández Bastida, Marta (2003). La implementación de un programa de gestión del conocimiento en una biblioteca universitaria. // Jornadas FESABID 2003: Los sistemas de información en las organizaciones: eficacia y transparencia. Barcelona: FESABID, 2003. 269-279. 